Bajopas Volume 2 Number 2 December, 2009

$$
\begin{aligned}
& \text { Bayero Journal of Pure and Applied Sciences, 2(2): } 1 \text { - } 8 \\
& \text { Received: April, } 2009 \\
& \text { Accepted: July, } 2009
\end{aligned}
$$

\section{THE NON-GENOMIC EFFECTS OF HIGH DOSES OF ROSIGLITAZONE ON CELL GROWTH AND APOPTOSIS IN CULTURED MONOCYTIC CELLS}

\author{
*S.A. Isa, L.S. Mainwaring, R. Webb, and A.W. Thomas \\ Centre for Biomedical Sciences, University of Wales Institute, Cardiff CF5 2YB, UK. \\ Correspondence author: sisa@uwic.ac.uk
}

\begin{abstract}
Peroxisome Proliferator-Activated Receptor gamma (PPARY) is a ligand-activated transcription factor which belongs to the nuclear hormone superfamily and has multiple pharmacological ligands called Thiazolidinediones (TZDs). TZDs are a class of drugs used in the treatment of type 2 diabetic patients. Rosiglitazone is one such TZD, and is used clinically to treat type 2 diabetes. In this study, the effect of Rosiglitazone on cell growth and apoptosis in cultured monocytic monomac 6 (MM6) cells was investigated. Over a 14 day period, MM6 cells were cultured in vitro and treated with $1 \mu M$

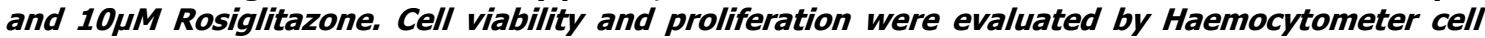
count and MTS assay respectively. Turbidity due to cell density was assessed spectrophotometrically. Apoptosis was determined by Caspase-Glo 3/7 assay. Expression of the endoplasmic reticulum (ER) stress-inducible protein sarco-endoplasmic reticulum $\mathrm{Ca}^{2+} \mathrm{ATPase}^{2} \mathrm{bb}$

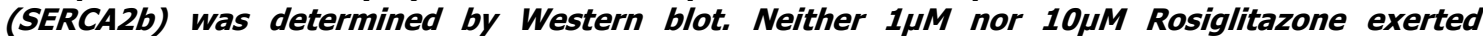
statistically significant inhibitory effects on cell proliferation, turbidity due to cell density, or cell viability ( $p>0.05$ in all cases). In contrast, Rosiglitazone induced increased apoptosis, but a

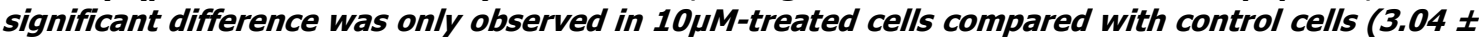
0.52 control; $p<0.05)$ while $1 \mu M$-treated cells showed a non-significant increase (1.50 \pm 0.06 control; $p>0.05)$. Meanwhile the expression of SERCA2b was up-regulated significant/y in cells treated for $>4$ hrs (e.g $2.45 \pm 0.06$ control at 24 hrs; $p<0.05$ ) with $10 \mu M$ Rosiglitazone. It was

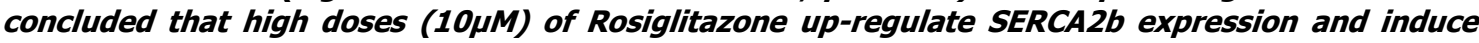
apoptosis of MMG cells by activating an ER stress response via a PPARY-independent mechanism. The therapeutic relevance of these observations is a matter for further investigations.
\end{abstract}

Key words: Rosiglitazone, PPARY, Monocytes, ER Stress, SERCA2b, Apoptosis

\section{INTRODUCTION}

Type 2 diabetes mellitus (T2D) is a disease condition characterised by defects in insulin action and secretion resulting from impaired beta cells secretory function. Insulin resistance results in notable decrease in glucose utilisation by the peripheral tissues with an increase in glucose production by the liver. The disease is found in almost every population worldwide. Epidemiological studies have shown that without effective prevention and control programmes, the prevalence of the disease will continue to rise globally (Alberti et al., 2007). No less than 171 million people live with diabetes worldwide in 2000 and this figure likely to increase to about 366 million by the year 2030 (Wild et al.,2004).

The thiazolidinediones (TZDs) are a class of oral drugs used for the management of type 2 diabetes mellitus. TZDs are synthetic ligands for the transcription factor Peroxisome Proliferator-Activated Receptor gamma (PPARY). Binding of TZDs to PPARY forms a heterodimer with Retinoid X Receptor (RXR) (Olefsky, 2000) that binds to the PPARY response element (PPRE) within the promoter of PPARy target genes, whose expression impacts upon different aspects of carbohydrate and lipid metabolism. Rosiglitazone, an example of TZD, is an anti-diabetic agent acting as a potent insulin sensitizer and is used clinically to enhance insulin-stimulated glucose uptake by tissues. Rosiglitazone has been shown to improve glycemic control (Raskin et al., 2001) and reduced glycated haemoglobin levels in patients with type 2 diabetes (Nissen et al., 2007).

Rosiglitazone has been shown to exert two types of effects: Genomic (PPARy-dependent) and Nongenomic (PPARy-independent) modes of actions. Thus, while it exerts anti hyperglycaemic effects by acting as ligands for PPARY, studies have shown that other effects may occur independent of PPARy. For example, our research group recently showed that Rosiglitazone induces PPARy-independent nongenomic effect such as sarco/endoplasmic reticulum calcium ATPase 2b (SERCA2b) inhibition after incubation for periods as brief as 5-30 minutes (Caddy et al., 2008a). However, after extended period of incubation (>24hrs), there was a compensatory upregulation of SERCA2b MRNA and calcium homeostasis was restored (Caddy et al., 2008a). $\mathrm{SERCA} 2 \mathrm{~b}$ is an enzyme responsible for translocating calcium from the cytosol to the lumen of the endoplasmic reticulum. Interestingly Zhong et al. (2001) reported that SERCA2b inhibition was related to the progression of type 2 diabetes (Zhong et al., 2001). The endoplasmic reticulum (ER) serves several important functions including post-translational modification, folding and assembly of newly synthesised proteins and calcium homeostasis. 
Of direct interest here is calcium homeostasis, as it relates to the pathophysiology of type 2 diabetes and its complications (Advani et al., 2004). Thus, this study investigated whether Rosiglitazone exerts adverse effects at a cellular level in vitro.

Disturbance of the ER functions (e.g. depletion of calcium from ER lumen) causes a condition collectively termed "ER Stress" (Kaufman et al., 2002). However, to continue to survive in ER stress environment, cells have developed a mechanism to overcome such stress called "ER Stress Response. ER stress responses (also known as unfolded protein responses or UPRs) involve the upregulation of genes containing ER stress response element (ERSE) and/or unfolded protein response element (UPRE) in their promoters. Examples of such genes are SERCA2b (Caspersen et al., 2000) and BiP (Araki et al., 2003). The effect of ER stress leading to the UPR activation has been linked to the development of insulin resistance and other metabolic diseases (Gregor et al., 2007).

Activation of PPARy by Rosiglitazone has been shown to inhibit cell growth by inducing apoptosis (Mao et al., 2007). This study focused on ER stress-mediated effects on cell growth, viability and apoptosis in MM6 cells. We show here that exposure of MM6 cells to high dose $(10 \mu \mathrm{M})$ of Rosiglitazone induced an increase in apoptosis. Cell viability was only marginally reduced (compared to control) from day 12 to 14 of MM6 cells incubation in $1 \mu \mathrm{M}$ and $10 \mu \mathrm{M}$ Rosiglitazone. Finally, treatment with Rosiglitazone induced up-regulation of the ER stress-inducible protein, SERCA2b.

\section{MATERIALS AND METHODS}

Materials. All reagents were purchased from SigmaAldrich (Poole, UK) unless otherwise stated. Cell permeabilization reagents and protein assay reagents were obtained from Harlan SERA-LAB Ltd (Loughborough, UK) and Bio-Rad Laboratories (Herts, UK) respectively. Rosiglitazone was obtained from GlaxoSmithKline (Uxbridge, UK). Mouse monoclonal primary anti SERCA2b antibody was purchased from Abcam (Cambridge, UK). HRP-Labelled Donkey antimouse IgG secondary antibody was purchased from New England Biolabs (Herts, UK). Cultured MM6 monocytic cells were obtained from the German Collection of MicroOrganisms and Cell Culture (Braunschwieg, Germany).

Cell Culture. Monocytic MM6 cells were cultured in Roswell Park Memorial Institute (RPMI) 1640 medium supplemented with $10 \%(\mathrm{v} / \mathrm{v})$ foetal calf serum, $1 \%(\mathrm{v} / \mathrm{v})$ penicillin $(50 \mathrm{IU} / \mathrm{ml}), 1 \%$ L-glutamine $(2 \mathrm{mM}), 1 \%(\mathrm{v} / \mathrm{v})$ non-essential amino acids and $1 \%(\mathrm{v} / \mathrm{v})$ OPI supplement. The cells were maintained at $37^{\circ} \mathrm{C}$ with $5 \% \mathrm{CO}_{2}$. The sub-cultured cells were maintained at $0.3-1.0 \times 10^{6}$ Cells $/ \mathrm{ml}$. Cells of passage number $<24$ were used in all experiments.

Incubation of Cells with Rosiglitazone. Cells were pooled all together and re-suspended in fresh medium. Two centrifuge tubes were used to spin down the cells

Apoptosis Assay. Apoptotic cells were determined using Caspase-Glo 3/7 Assay. This was performed in accordance with the manufacturer's instructions.

Statistical Analysis. Data are expressed as the mean of three independent experiments and were compared using Student's $t$-test. $p<0.05$ was considered statistically significant. using a Harrier Centrifuge 15/18. After spinning, supernatant was discarded and the cells were resuspended in fresh medium. $0.1 \%$ DMSO $(\mathrm{v} / \mathrm{v}), 1 \mu \mathrm{M}$ or $10 \mu \mathrm{M}$ Rosiglitazone (dissolved in DMSO to $\leq 0.1 \% \mathrm{v} / \mathrm{v}$ ) was then added to the samples for incubation over the subsequent 1-14 days.

Preparation of Total Protein Extracts and Western Blot Analysis. After incubation with DMSO \pm Rosiglitazone, cultured cells $\left(5 \times 10^{6} \mathrm{cell} / \mathrm{s} / \mathrm{ml}\right)$ were placed in a sterile centrifuge tube and spun down at 300RCF for 10 minutes. The supernatant was removed and cells were washed in $10 \mathrm{ml}$ ice cold PBS buffer. The washing step was repeated twice. Cells were then lysed for 30 minutes on ice in $112 \mu \mathrm{l}$ of RIPA buffer [ $(100 \mathrm{mM} \mathrm{NaCl}$; $10 \mathrm{mM}$ Tris $\mathrm{HCl} ; 2 \mathrm{mM}$ EDTA; $0.5 \%$ (w/v) sodium

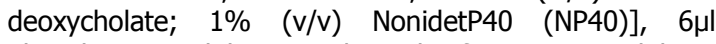
phosphatase inhibitor and $12 \mu \mathrm{l}$ of protease inhibitor cocktail. Sample lysates were sonicated for few seconds on ice to shear DNA to reduce sample viscosity. Protein concentrations of whole cell lysates were then determined using BioRad DC Protein assay according to the manufacturers' instructions (BioRad Laboratories, Herts, UK).

For western blot experiments, Loading buffer was then added to each lysate and then heated for about 5 minutes in a waterbath. Portions ( $50 \mu \mathrm{g}$ each) of heated protein samples were then loaded into separate wells of the gel. To the first well, MagicMark molecular weight marker was loaded and MultiMark pre-stain molecular marker in the last well. The gel was run at 200 volts for 50 minutes at room temperature. After electrophoresis, samples were transferred on to nitrocellulose membranes. Each membrane was then incubated in primary antibody for 1 hour at room temperature (1:500 dilutions). The membrane was washed 3 times at 5 minutes interval using TBS/Tween20. The membrane was subsequently incubated with HRP-conjugated secondary antibody (1:1000 dilutions) for 3 hours at room temperature before addition of enhanced chemiluminescence-coupled HRP substrate, and visualisation using UVP VisionWorksLs, version 5.54.

Measurement of Cell Viability. Optical density (turbidity) and Trypan blue exclusion methods were used to measure the number of viable cells in the samples. A light scattering technique was used to monitor the concentration of cells in Rosiglitazone treated samples; the amount of light absorbed by the suspension of cells was measured using spectrophotometer at $600 \mathrm{~nm}$. A calibration curve was constructed to show that $\mathrm{OD}_{600}$ is proportional to cell number. Trypan blue was used to assess the number of viable cells in the sample. Live cells possessed an intact cell membrane which excludes the dye. However, dead cells were stained blue by the dye. Haemocytometry was then used to count the viable cells. Cell Proliferation Assay. The actively dividing cells in the Rosiglitazone treated samples were assessed using CellTiter 96 Aqueous One Solution cell proliferation assay (MTS assay) according to the manufacturer's instructions.

\section{RESULTS}

Effect of Rosiglitazone on Cell Proliferation. To examine the effect of Rosiglitazone on cell proliferation, MM6 cell proliferation rate was analyzed by MTS assay. The results show that $1 \mu \mathrm{M}$ and $10 \mu \mathrm{M}$ Rosiglitazone do not exert a statistically significant inhibitory effect on cell proliferation ( $p>0.05$ in both cases) (Figure 1). 


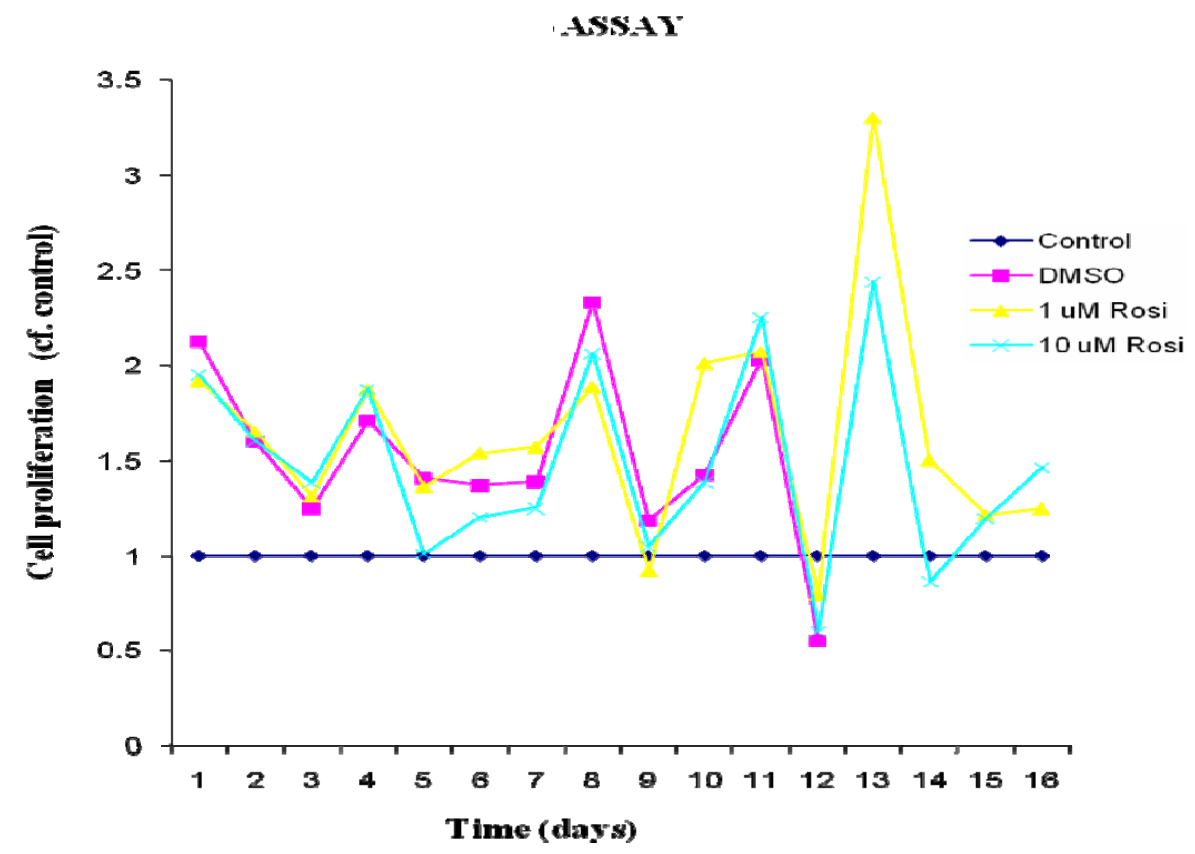

Figure 1: The effect of Rosiglitazone on cell proliferation of MM6 cells treated with $0.1 \% D M S O, 1 \mu M$ and $10 \mu M$ Rosiglitazone.

\section{Effect of Rosiglitazone on Cell Viability}

To examine the effect of Rosiglitazone on MM6 cell viability, a direct cell count using haemocytometer was performed. Although cell viability was marginally reduced (compared to control) from day 12 to 14 of the incubation in $1 \mu \mathrm{M}$ or $10 \mu \mathrm{M}$ Rosiglitazone, the effect of both $1 \mu \mathrm{M}$ and $10 \mu \mathrm{M}$ Rosiglitazone did not reach statistical significance (Figure 2).

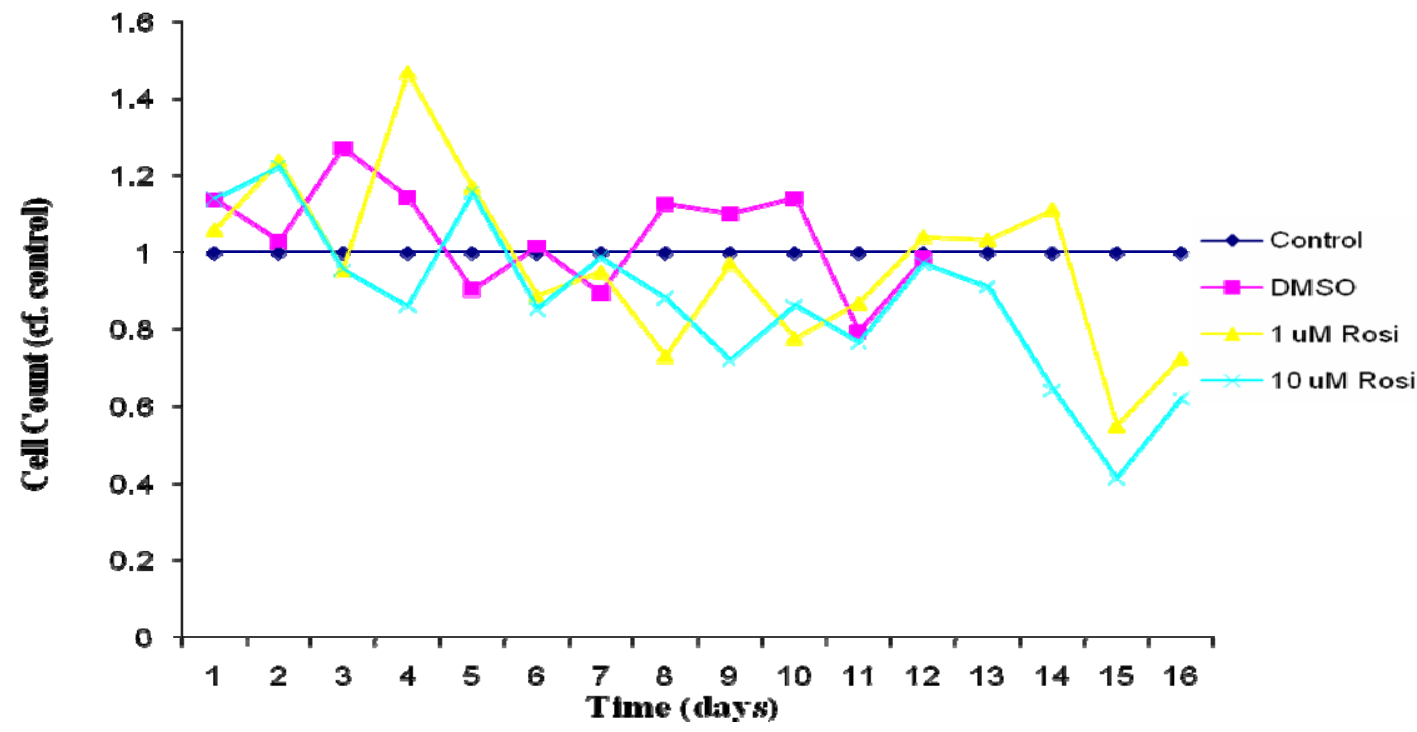

Figure 2: The effect of Rosiglitazone on cellular viability of MM6 cells treated with $0.1 \%$ DMSO, $1 \mu \mathrm{M}$ and $10 \mu \mathrm{M}$ Rosiglitazone. 
Bajopas Volume 2 Number 2 December, 2009

\section{Effect of Rosiglitazone on Turbidity due to Cell Density}

Optical density measurements at $600 \mathrm{~nm}$ were employed to determine the amount of light scattered by the suspension of cells. As the optical density or turbidity of a suspension of cells is directly related to cell number (Fig. 3a), our optical density results allow us to conclude that no significant difference (Fig.3b) in cell density is seen in $1 \mu \mathrm{M}$ and $10 \mu \mathrm{M}$ Rosiglitazone treated samples compared to control ( $p>0.05$ in both cases).

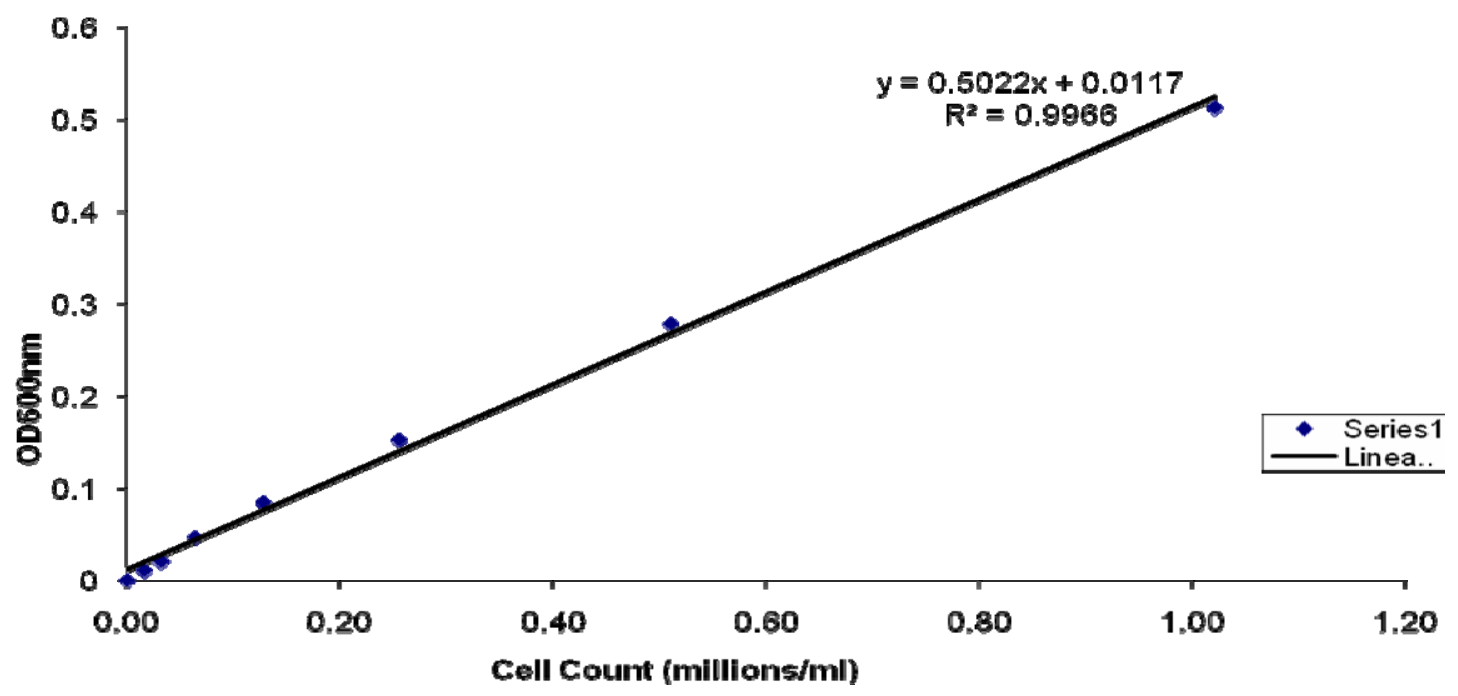

Figure 3a: Optical Density Calibration curve. The calibration curve shows that ${ }^{O} D_{600}$ is proportional to cell number.

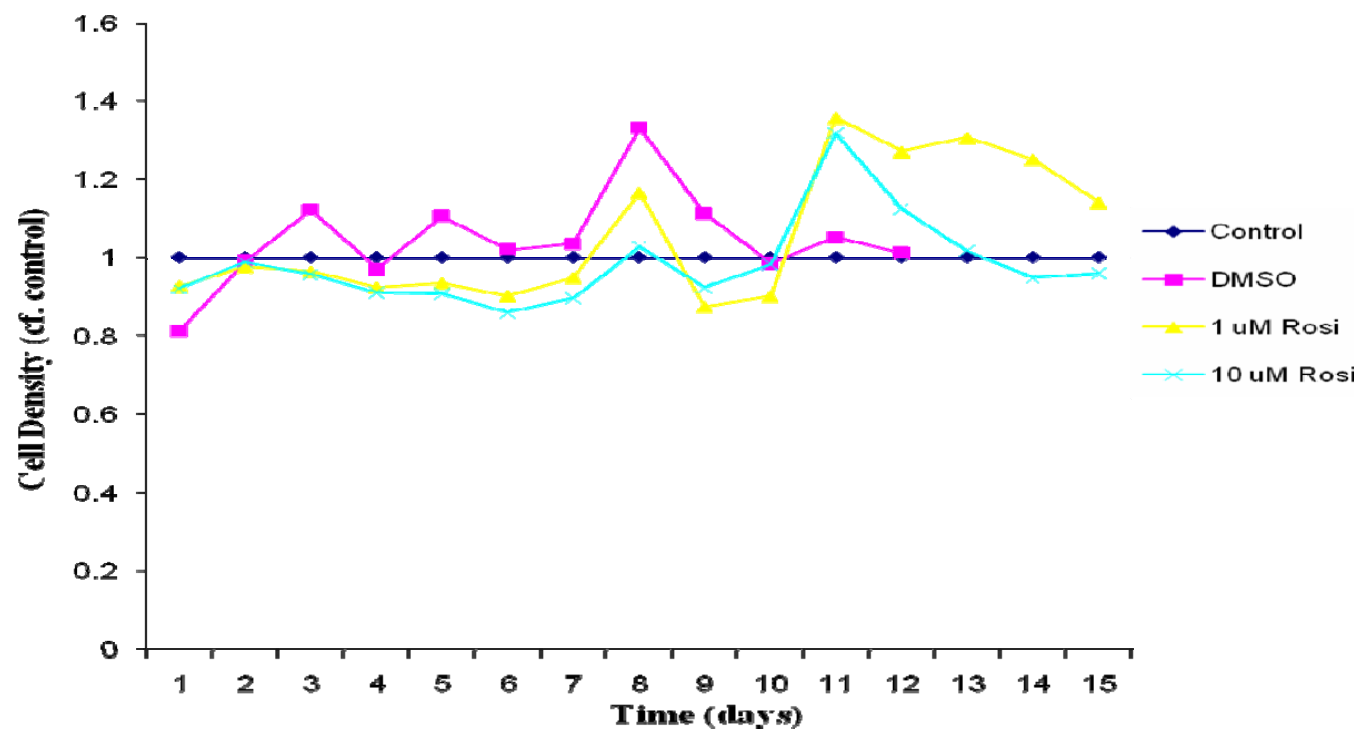

Figure 3b: Effect of Rosiglitazone on Turbidity due to Cell Density for MM6 cells treated with $0.1 \%$ DMSO, $1 \mu M$ and $10 \mu \mathrm{M}$ Rosiglitazone.

Expression of an ER Stress-Inducible protein (SERCA2b) in Rosiglitazone Treated MM6 cells

To investigate the effect of $10 \mu \mathrm{M}$ Rosiglitazone on the expression of SERCA2b protein, a Western Blot analysis was performed. SERCA2b was detected by the Western Blot as approximately $105 \mathrm{KDa}$ band (Fig.
Rosiglitazone treated cells. The result (Fig. 4b) showed an increase (approx. x2.5 fold) in expression of this protein in the MM6 cells treated for $>4 \mathrm{hrs}$ with $10 \mu \mathrm{M}$ Rosiglitazone. Thus, it can be concluded that Rosiglitazone induces an ER stress response in these cells. 


\section{Time (h)}

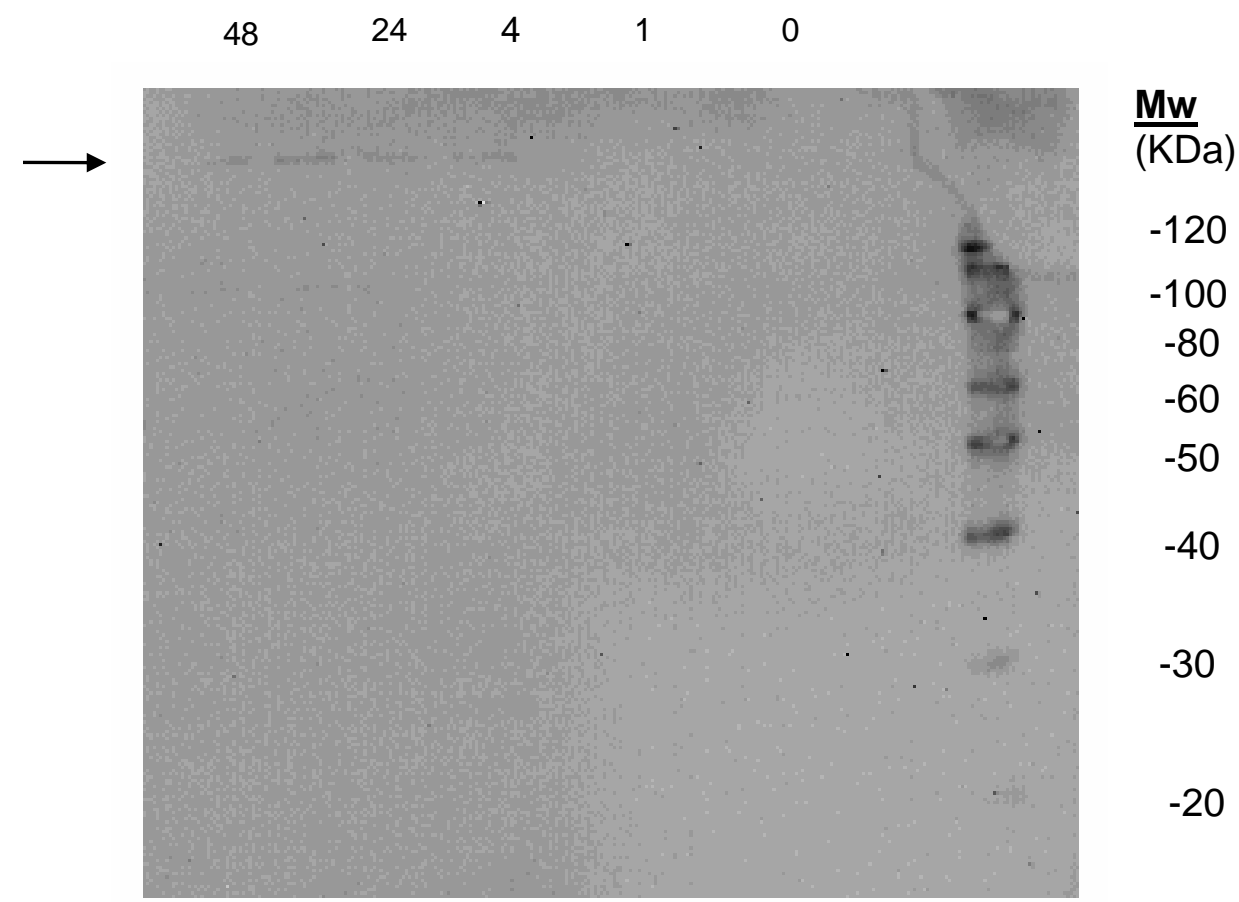

Figure 4a: Western Blot analysis of SERCA2b in MM6 cells total protein lysate. SERCA2b was detected using antiSERCA2b primary antibodies and HRP-labelled secondary antibodies. Arrow indicates 105KDa molecular weight bands, which correspond to SERCA2b. Image is representative of three separate experiments

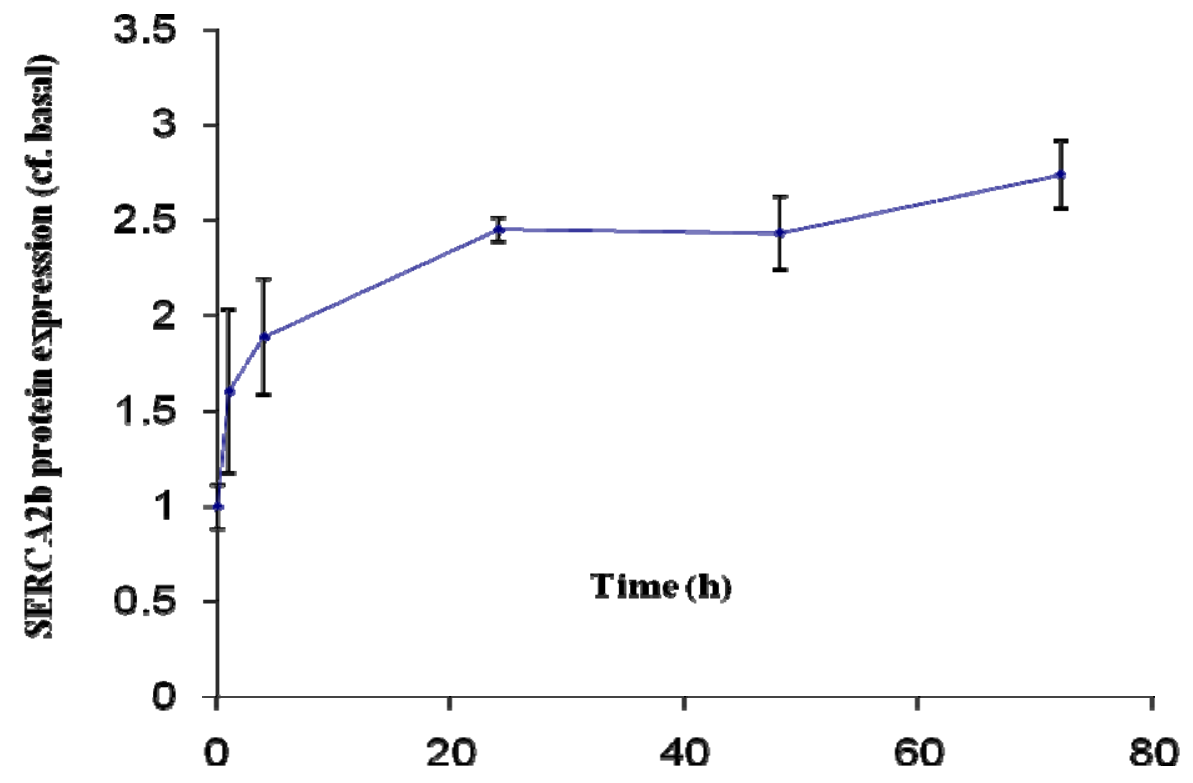

Figure 4b: Densitometric analysis of SERCA2b protein expression in MM6 cells treated with 10 $\mu M$ Rosiglitazone. $>4$ hrs incubation with $10 \mu \mathrm{M}$ Rosiglitazone showed an increase (approx. x2.5 fold) in expression of SERCA2b. Data are from 3 separate experiments.

\section{Induction of MM6 Apoptosis by Rosiglitazone}

As ER stress responses can lead to either restoration of normal cell physiology or to apoptosis, MM6 cells were treated with $1 \mu \mathrm{M}$ and $10 \mu \mathrm{M}$ Rosiglitazone for 14 days and subsequent apoptosis was assessed by Caspase-Glo 3/7 Assay. Although 14 days exposure to Rosiglitazone induced increases in apoptosis in MM6 cells, the results (Fig. 5) showed a significant difference only in $10 \mu \mathrm{M}$ treated cells compared with control cells $(3.04 \pm 0.52$ control; $p<0.05$ ) while $1 \mu \mathrm{M}$ Rosiglitazone treated cells showed only a non-significant increase $(1.50 \pm 0.06$ control; $p>0.05$ ). 


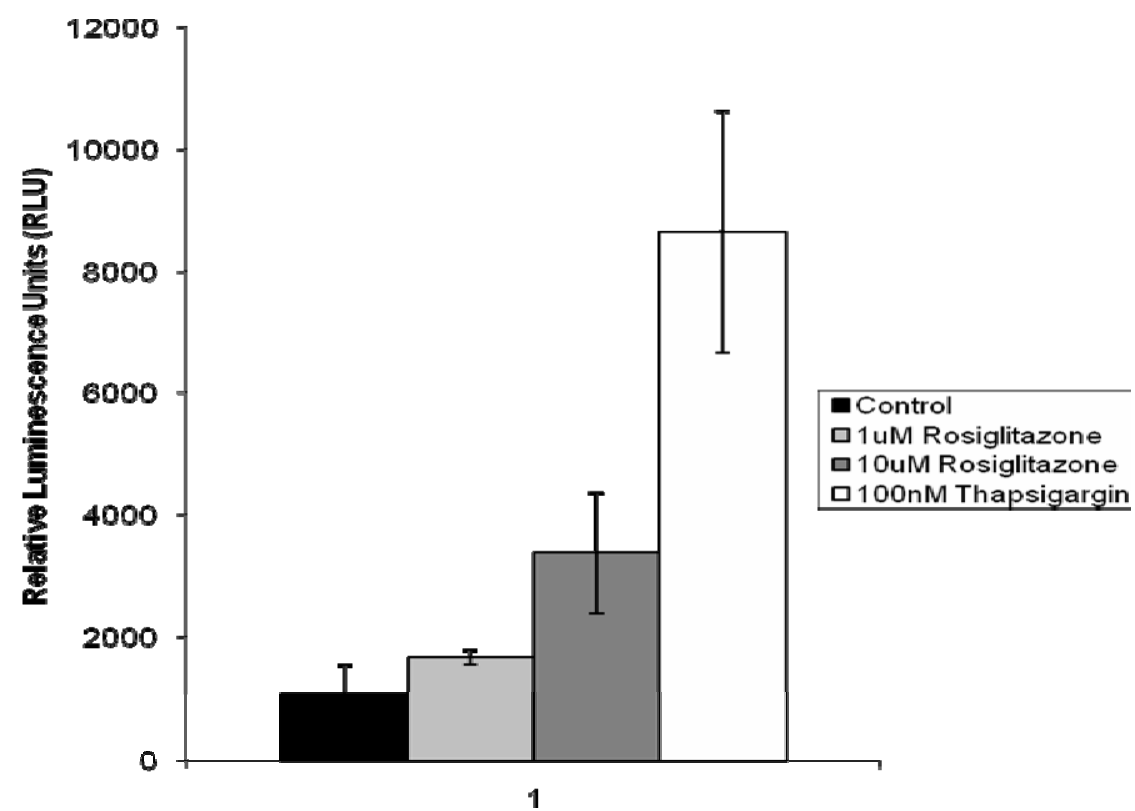

Figure 5: Effect of Rosiglitazone on Apoptosis of MM6 cells by Caspase-Glo 3/7 assay. Data are expressed as the mean of three independent experiments (NB: Cells were treated with thapsigargin (Tg) for only four days, as longer duration of $\mathrm{Tg}$ treatment proved cytotoxic).

\section{DISCUSSION}

The discovery of TZDs as potent insulin sensitizers in diabetes patients has generated substantial interest into their pharmacological actions. The molecular mechanisms by which TZDs exert their actions are quite complex and studies have shown that they may have differential effects in some tissues (Camp et al., 2000). However, TZDs have been shown to exert two types of effects: PPARY-dependent and PPARYindependent modes of action. Thus, although Rosiglitazone exerts anti-hyperglycaemic effects by acting as a ligand for the nuclear receptor PPARY, studies have shown that effects such as those observed in the present study may occur independent of PPARY (so called non-genomic effects). It should be noted that disruptions in calcium homeostasis, which have been associated with the pathophysiology of type 2 diabetes and its complications (Zhong et al., 2001), have been linked to the non-genomic effects of PPARy ligands in this and previous studies. For example, a recent study in our laboratory has shown that short-term (<30 mins) incubation with Rosiglitazone inhibits SERCA2b via a PPARYindependent (non-genomic) manner (Caddy et al., 2008a). However, after extended period of incubation (>24hrs), there was a compensatory up-regulation of SERCA2b and restoration of calcium homeostasis in monocytic cells (Caddy et al., 2008a). As mentioned earlier, the endoplasmic reticulum (ER) serves several important functions including calcium homeostasis. Recently, Rosiglitazone has been linked to disruption of ER (Caddy et al., 2008b), which may be suggested to lead to induction of an ER stress response. ER stress responses cause up-regulation of ER stressinducible genes such as SERCA2b (Caspersen et al., 2000). In this study, SERCA2b was detected by western blot as approximately $105 \mathrm{KDa}$ band in the total protein lysate of Rosiglitazone-treated MM6 cells.

The results showed an increase (approx. x2.5 fold) in expression of the 105KDa (Fig. 4b) protein in MM6 cells treated for $>4$ hrs with $10 \mu \mathrm{M}$ Rosiglitazone. Rosiglitazone has also been shown to inhibit cell growth by inducing apoptosis (Mao et al., 2007), and ER stress responses can lead to apoptosis (Di Sano et al., 2006; Szegedi et al., 2006); therefore this study focused on ER stress-mediated inhibition of cell growth and viability in MM6 cells. Although cell viability was marginally reduced (compared to control) from day 12 to 14 of the incubation in $1 \mu \mathrm{M}$ and $10 \mu \mathrm{M}$ Rosiglitazone, the effect in both cases did not reach a statistical significance $(p>0.05)$. The results suggest that up-regulation of SERCA2b helps to largely restore normal intracellular calcium homeostasis. While incubation of MM6 cells with $10 \mu \mathrm{M}$ Rosiglitazone leads to approximately 3 fold increases in apoptosis, this effect is much less marked than that seen with the classical SERCA2b inhibitor Thapsigargin (Tg). Moreover, 1uM Rosiglitazone (which approximated to the concentration seen in T2D patients in vivo (Niemi et al., 2003)) does not have a significant effect in this regard.

In conclusion, results from this study show an increase (approx. x2.5 fold) in expression of SERCA2b in MM6 cells treated with Rosiglitazone. This up-regulation of SERCA2b may be important in maintaining calcium homeostasis in the cells of patients undergoing Rosiglitazone therapy. Since TZDs have been shown in several studies to be associated with unfavourable side effects (Soroceanu et al., 2004; Nissen et al., 2007; Singh et al., 2007; Sarafidis 2008), there is a need to identify natural and/or synthetic PPARy ligands that have the ability to 
Bajopas Volume 2 Number 2 December, 2009

exert PPARy-dependent anti-hyperglycemic effects, without also inducing deleterious side-effects. There is also the need to investigate whether the mechanism via which Rosiglitazone exerts its effect in monocytes also occur in other cell types, like skeletal muscle and cardiomyocytes. While pharmacological doses of Rosiglitazone (Rosiglitazone has been shown to exist in the plasma at concentration approximately $1 \mu \mathrm{M}$, (Niemi et al., 2003)) did not significantly induce

\section{REFERENCES}

Advani A, Marshall SM and Thomas TH (2004). Impaired Neutrophil Store-Mediated Calcium Entry in Type 2 Diabetes. European Journal of Clinical Investigation. 34:43-49.

Alberti KGM, Zimmet P and Shaw J (2007). International Diabetes Federation: A Consensus on Type 2 Diabetes Prevention. Diabetic Medicine. 24(5):452-463.

Araki E, Oyadomaris S and Mori M (2003). Impact of Endoplasmic Reticulum Stress Pathway on Pancreatic $\beta$-cells and Diabetes Mellitus. Experimental Biology and Medicine. 228(10):1213-1217.

Caddy J, Singh N, Atkin L, Ahluwalia M, Roberts A, Lang D, Thomas AW and Webb R (2008a). Rosiglitazone Transciently Disturbs Calcium

Caspersen C, Pedersen PS and Treiman M (2000). The Sarco/Endoplasmic Reticulum CalciumATPase 2b Is an Endoplasmic Reticulum Stress-inducible Protein. The Journal of Biological Chemistry. 275(29):22363-22372.

Di Sano F, Ferraro E, Tufi R, Achsel T, Piacentini M and Cecconi F (2006). Endoplasmic Reticulum Stress Induces Apoptosis by an Apoptosome-dependent but Caspase 12independent Mechanism. Journal of Biological Chemistry. 281(5):2693-2700.

Gregor MG and Hotamisligil GS (2007). Adipocyte Stress: The Endoplasmic Reticulum and Metabolic Disease. Journal of Lipid Research. 48:1905-1914.

Kaufman RJ, Scheuner D, Schroder M, Shen X, Lee K, Liu CY and Arnold SM (2002). The Unfolded Protein Response in Nutrient Sensing and Differentiation. Nature Review Molecular Cell Biology. 3:411-421.

Mao SL, Wei CC, Xia B and Ya DW (2007). Activation of Peroxisome Proliferator-Activated Recepor Gamma Inhibits Cell Growth via apoptosis and Arrest of the Cell Cycle in Human Colorectal Cancer. Journal of Digestive Diseases. 8(2):82-88.

Niemi M, Beckman JT, Granfors M, Laitila J, Neuvonen $M$ and Neuvonen PJ (2003). Gemfibrozil Considerably Increases the Plasma Concentrations of Rosiglitazone. Diabetologia. 46:1319-1323.

Nissen SE and Wolski K (2007). Effect of Rosiglitazone on the Risk of Myocardial Infarction and Death from Cardiovascular Causes. The New England Journal of Medicine. 356:2457-2471. apoptosis, high doses of Rosiglitazone $(10 \mu \mathrm{M})$ could inhibit cell growth by inducing apoptosis via the ER stress response pathway. Since ER stress responses have been linked to the development of insulin resistance and other metabolic diseases (Gregor et al., 2007), discoveries in this aspect indicates the importance of ER stress responses in type 2 diabetes, and suggest that they could be a target for the management of the disease.

Homeostasis in Monocytic Cells. Biochemical and Biophisical Research Communications. 366(1):149-155.

Caddy J, Thomas AW, Atkin L, Roberts A.W, Lang D, and Webb R (2008b) Rosiglitazone Induces Unfolded Protein Responses, but not Apoptosis, in Monocytes and Vascular Smooth Muscle Cells - poster presentation at the $3^{\text {rd }}$ "Controversies in Obesity, Diabetes and Hypertension" Conference, Barcelona, Spain.

Camp HS, Li O, Wise SC, Hong $\mathrm{YH}$, Frankowski CL, Shen X, Vanbogelen R and Leff T (2000). Differential Activation of Peroxisome Proliferator Activated Receptor gamma by Troglitazone and Rosiglitazone. Diabetes. 49(4):539-547.

Olefsky JM (2000). Treatment of Insulin Resistance with Peroxisome Proliferator-Activated Receptor y Agonist. The Journal of Clinical Investigation. 106(4):467-472.

Raskin P, Rendell M, Riddle MC, Dole JF, Freed MI and Rosenstock J (2001). A Randomised Trial of Rosiglitazone Therapy in Patients with Inadequately Controlled Insulin-Treated Type Diabetes. Diabetes Care. 24:1226-1232.

Sarafidis PA (2008). Thiazolidinedione Derivatives in Diabetes and Cardiovascular Disease: An Update. Fundamental \& Clinical Pharmacology. 22(3):247-264.

Singh S, Loke YK and Furberg CD (2007). Thiazolidinediones and Heart Failure: A Teleo-Analysis. Diabetes Care. 30:21482153.

Soroceanu MA, Miao D, Bai X, Su H, Goltzman D and Karaplis AC (2004). Rosiglitazone Impacts Negatively on Bone by Promoting Osteoblast/Osteocyte Apoptosis. Journal of Endocrinology. 183:203-216.

Szegedi E, Logue SE, Gorman AM and Samali A (2006). Mediators of Endoplasmic Reticulum Stress-Induced Apoptosis. EMBO Reports. 7(9):880-885.

Wild S, Roglic G, Green A, Sicree R and King H (2004). Global Prevalence of Diabetes: Estimates for the Year 2000 and Projections for 2030. Diabetes Care. 27(5):1047-1053.

Zhong Y, Ahmed S, Grupp IL and Matlib MA (2001). Altered SR Protein Expression Associated with Contractile Dysfunction in Diabetic Rat Hearts. American Journal of Physiology, Heart Circulation Physiology. 281:H1137H1147. 